\title{
Based on the Old Table of the Application of Dynamic Segmentation Technology Image Processing System
}

\author{
Chao Wang, Jun Zheng, and Jianhua Cai
}

\begin{abstract}
Watt hour meter recovery is a heavy and intensity task, so an automated system for recycling old Watt hour meter comes into being. The system through a specially developed image recognition program identifies Watt hour meter readings. Image processing in the system is particularly important. The image processing includes Locating object, object segmentation and object recognition, the ability to split out the object to be identified is a precondition of the object recognition. In this paper, we introduce the image processing in the old table recovery of automation system and its application, compare the global threshold segmentation and dynamic threshold segmentation to overcome the effects of uneven attention.
\end{abstract}

Index Terms-Watt hour meter recovery, image processing, image segmentation.

\section{INTRODUCTION}

Image preprocessing, also called image restoration, involves the correction of distortion, degradation, and noise introduced during the imaging process. Currently, because the artificial old watt-hour meter is a heavy task, working strength is big, the low efficiency of a work, with the development of technology, a kind of automation system for the old table recycling arises at the historic moment. And image processing function is particularly important in the system, this system through specialized development of image recognition process, identification of watt-hour meter readings, implementation of watt-hour meter information available, and can split out to identify the target is a prerequisite for target recognition.

Image segmentation is a key technique in image processing, has always attached great importance to by the people, has thousands of segmentation algorithm is proposed. But because there is no general theory of segmentation, the segmentation algorithm proposed is mostly aimed at specific problems, not a general image segmentation algorithm is suitable for all images. In addition, also not make choice suitable segmentation algorithm, it gives the application of image segmentation technology bring a lot of practical problems.

This paper first introduced the common method to split, and then introduces the method used in this project.

Manuscript received September 24, 2014; revised December 5, 2014. This work was financially supported by the National High Technology Research and Development Program of China (863 Program 2013AA01A211).

The authors are with the Computer Center, East China Normal University 200062 Shanghai, China (e-mail: sf5415@qq.com).

\section{The Common Method to Image Segmentation}

During the past 10 years, there has been a significant research increase in image segmentation. To study a specific object in an image, its boundary can be highlighted by an image segmentation procedure. The current image segmentation method is mainly divided into threshold segmentation method, edge detection method, region extraction method, and combining the theory of specific tools of segmentation method of this a few kinds big. The goal of image segmentation is to cluster pixels into salient image regions. [1]

\section{A. Threshold Segmentation Method}

Take threshold of gray image segmentation is to determine an image gray value range of gray threshold, then the image of each pixel gray value are compared with the threshold value, and according to the comparison results of corresponding pixels can be divided into two kinds, the two types of pixel generally belong to the image of two types of area, so as to achieve the goal of segmentation. In the method to determine an optimal threshold is the key to segmentation can achieve better effect, most of the existing threshold segmentation algorithms are concentrated on the threshold value to determine the research. Fig. 1 shows the different results of globally and locally threshold method.

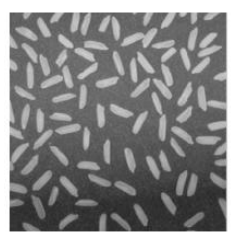

Original Image

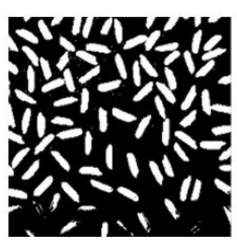

Globally Thresholded Locally Thresholded
Fig. 1. Results of globally threshold and locally threshold.

At present many new threshold segmentation method is proposed, based on quantization histogram is presented as Yanxue Jiang maximum entropy threshold processing algorithm [2], the histogram quantization after using maximum entropy threshold processing algorithm, greatly reduce the amount of calculation. Xue Jinghao, Yu-jin Zhang et al., [3] between posterior cross entropy is put forward based on the categories of threshold segmentation algorithm, starting from the differences between classes of target and background, estimated by the Bayesian formula pixel belongs to two kinds of target and background region of the a posteriori probability, and then search the two types of area between the posterior probability of maximum cross entropy, Yuyong Deng people use of image segmentation method 
based on minimum energy [4], the use of the energy histogram threshold segmentation to select. Ren Mingwu put forward for constructing a fringe pattern based on the histogram method [5], the segmentation threshold is affected by noise and the edge of the reduced to minimum. Zhi-yong he put forward a new algorithm, the algorithm based on two-dimensional histogram of the thought of linear threshold segmentation, using two-dimensional pixel point information directly establish threshold linear intercept histogram; Then apply Otsu guidelines for the one-dimensional histogram optimal intercept threshold, and the application of the threshold and the two-dimensional information [6], complete image segmentation algorithm of this method are compared with those of the traditional two-dimensional Otsu method and analysis. All these algorithms no matter what method, combined with what tools, the basic idea is consistent, is to seek the best segmentation threshold.

\section{B. The Segmentation Method Based on Edge}

The segmentation method based on edge, according to the different methods of edge detection using edge detection method includes the following categories: roughly based on local image function method, the multi-scale method, image filtering method, the method based on reaction - diffusion equation, multi-resolution method, based on boundary curve fitting method, the state space search method, dynamic programming method, boundary tracking method, hough transform method, etc. As huan-sheng song [7] multi-scale ridge edge method is proposed, the method of wavelet decomposition, image 2 schedule and then calculate the multi-scale ridge edges of binary scale space and intensity, finally through the ridge edge tracking, filtering and inverse discrete wavelet transform, the segmentation results are obtained. Zhang Jing [8] put forward the line scan space band-pass filtering method, is on the basis of summarizing the predecessors' theory and experimental results put forward a new method of edge detection, automatic tracking of TV image recognition has a good effect.

\section{Based on Region Segmentation Method}

The essence of the regional segmentation is to have a certain similarity pixel connected together, so as to form the final segmented regions. In this kind of method, if starting from the map, according to the regional attribute characteristics consistent criterion, decided each as regional identity, forming region diagram, this is often called the region growing segmentation method; If starting from like yuan, according to the regional attribute characteristics consistent criterion, the property is close to the connected pixels, gathered to the growth of the region segmentation method for area; If the comprehensive utilization of the above two methods, it is splitting - merging method. Hu Zhengping et al. [9] is put forward based on region evolved adaptive region growing image segmentation algorithm has high precision. In the process of image preprocessing, first by anisotropic filtering algorithm to filter slices, to remove the noise of the image at the same time avoid the fuzzy of border area; Then introduces a new regional energy representation model, and gives the iteration form of evolution, in the process of regional growth, gradually increase the threshold of regional growth, based on the energy function of dynamic optimization to optimal segmentation result; The final precision segmentation using active contour model, precise and smooth target contour segmentation. Ning-ning Liu put forward such as interactive image segmentation method based on the agent machine model [10], the agent machine is to achieve a specific function module, interface and reporting interface implementation with the operator control through the interaction, this method is especially suitable for medical image segmentation. Mr. Liu et al. [11] proposed a sequence image fusion algorithm based on region segmentation. First of all, the sequence of the infrared image is divided into three different feature regions, the target region and background region and the gray area, and the segmentation results map to visible light image. Then, using multi-scale geometric analysis tools under non sampling contourlet transform (NSCT) effectively extract the characteristics of image features, according to the characteristics of different areas in the NSCT domain design different fusion rules, sequence image fusion algorithm based on region segmentation is not only able to retain fused image is more comprehensive and abundant background information, also can more effectively and accurately extract the image characteristics of the target. The algorithm is superior to the traditional rules of fusion algorithm based on pixel and window, image fusion algorithm is an effective and feasible.

\section{The Method of Combining the Theory of Specific Tools}

So far there is no general image segmentation theory of itself. In recent years, with each course many new theory and new method is put forward, also put forward a lot of people with certain theories, methods and tools with the combination of segmentation techniques. Such as segmentation technology based on mathematical morphology, image segmentation method based on fuzzy technique, image segmentation method based on artificial neural network technology, etc.

Watt-hour meter in this project the position of the LCD screen are relatively consistent, so the goal of image segmentation is probably determined. Due to the light source is hard to adjust to a very uniform, so by adopting appropriate to the specific circumstances segmentation algorithm is the key to success. In view of the particularity of this project in part to choose the dynamic threshold segmentation, image segmentation in order to overcome the influence of uneven illumination in the image preprocessing before image segmentation.

\section{Dynamic IMAge Segmentation}

\section{A. Image Preprocessing}

Smooth mesh is mainly to prepare for the following Dynamic Segmentation, the main purpose is not to remove noise. This project choose average filtering in order to meet the demand. Linear filter, median filter, also known as the main method used in the bounded domain average method. The basic principle of linear filtering is average instead of the original image in each pixel value, is dealing with the current pixel $(x, y)$, choose a template, this template is composed of 
the neighbor number of pixels, strives for the average of all pixels in the template, and then gives the mean to the current pixel point $(x, y)$, as a processing after the image gray scale on which a $\mathrm{g}(x, y)$, namely a $\mathrm{g}(x, y)=1 / \mathrm{m} \sum f(x, y)$, $\mathrm{m}$ for this template contains the current pixel, the total number of pixels. With average filtering it exists inherent defects, that is, it cannot protect the image detail well, in the image denoising also destroyed the details of the image, so that the image is blurry, but this problem does not affect the project, because the average smooth mesh is mainly to prepare for the following Dynamic Segmentation, rather than to remove noise.

\section{B. The Dynamic Segmentation}

Dynamic Segmentation is a computer-based database management system using the principles of relational algebra and topological integrity to minimize error and maximize efficiency of map analysis and display. Linear features are stored as nodes and arcs and provide only the minimum of geometric properties for proper display on maps. When these linear features, such as roads and railways, have multiple attribute tables, Dynamic Segmentation is used to query and display such attributes.

Due to the particularity of the use of occasions, after waiting for the part of the image segmentation is known, and therefore FenGeGan only interested in the part of the image due to specific problems to overcome the uneven lighting.

As Brennan and Cadkin [12] describe in their article that, Dynamic Segmentation is the process of transforming linearly referenced data (also known as events) that have been stored in a table into features that can be displayed and analyzed on a map. There are two data requirements for performing Dynamic Segmentation. First, each event in an event table must include a unique identifier and its measurement along a linear feature. Second, each linear feature (route) must have a unique identifier and a measurement system stored with it. In the dynamic threshold segmentation method, dynamic threshold value is relative to the conventional fixed threshold, namely under the global optimal criterion, first determine the threshold to one (or more), the threshold has a certain ability to resist noise, based on this threshold, and use the location of the point of division grayscale distribution characteristics of the image point around, adjusted, the threshold is the dynamic threshold. Shall have a $g(o)$ as the source image pixels of the pixel value, $g(t)$ for the average image, the corresponding pixel values are for the delta set to a constant. If $g(o)<=g(t)$ - the delta is corresponding to the darker areas, if $g(o)>=g(t)+$ delta is corresponding to a LAN. LCD screen font than the background darker, we extract only meet the conditions of $\mathrm{g}$ $(o)<=g(t)$ - the delta area.

\section{CONCLUSION}

Image segmentation can be applied in variety of different field, such as pattern recognition, image compression, and image retrieval. In imaging science, image processing is any form of signal processing for which the input is an image.

This paper introduces the image processing in the old table recovery of automation system, the application of the system through specialized development of image recognition program, identify the watt-hour meter reading. Related content, this paper mainly discusses the image segmentation pretreatment before the first discusses the image segmentation and are suitable for dynamic threshold segmentation through the experiment of smooth size, then compared the global threshold segmentation and dynamic threshold segmentation to overcome the effects of uneven attention. Supporting both point and linear attributes, Dynamic Segmentation enables monitoring of incidents occurring at points. Any application involving linear features can benefit from using the functionality provided by dynamic segmentation. It is concluded that the dynamic threshold is more suitable for the project environment.

\section{REFERENCES}

[1] D. L. Pham, C. Xu, and J. L. Prince, "Current methods in medical image segmentation," Annual Review of Biomedical Engineering, vol. 2, pp. 315-337, Aug. 2000.

[2] Y. Jiang and X. Ye, "Based on the quantitative image histogram threshold processing algorithm of maximum entropy," Journal of Pattern Recognition and Artificial Intelligence, vol. 11, no. 3, pp. 352-258, 2011.

[3] J. Xue and Y. Zhang, "Based on the categories of posterior cross threshold algorithm for segmentation between," Chinese Journal of Image and Graphics, vol. 10, no. 6, pp. 111-114, 2011.

[4] Z. Chen and R. Zhou, "Image segmentation algorithm based on minimum energy," Play Arrow and Journal of Guidance, vol. 27, no. 4, pp. 333-335, 2012.

[5] M. Ren, J. Yang, and H. Sun, "A new method of histogram based on the pattern of the edge structure," Journal of Computer Research and Development, vol. 38, no. 8, pp. 972-976, 2011.

[6] Z. He, L. Sun, and L. Chen, "Based on Otsu guidelines and linear intercept histogram threshold segmentation," Optical Precision Engineering, vol. 20, no. 10, pp. 2315-2323, 2012.

[7] H. song, C. Liu, and C. Wu, "Multi-scale edge ridge edge and its application in image target segmentation," Journal of Automation, vol. 25 , no. 6 , pp. $12-15,2012$.

[8] J. Zhang and H. Wang, "An image segmentation method of edge detection," Journal of Optics, vol. 27, no. 5, pp. 24-26, 2011.

[9] Z. Hu and Mellisa, "Regional evolution adaptive precision region growing image segmentation algorithm," Journal of systems Engineering and Electronics, vol. 29, no. 6, pp. 854-857, 2011.

[10] N. N. Liu and T. Jie, "Interactive image segmentation method based on regional characteristics and its application," Journal of Software, vol. 12, no. 3, pp. 10-18, 2011.

[11] K. Liu and L. Guo, "A sequence image fusion algorithm based on region segmentation," Infrared and Laser Engineering, vol. 6, no. 3, pp. 553-558, 2009.

[12] J. Cadkin and P. Brennan, "Dynamic segmentation in ArcGIS," ArcUser, pp. 52-54, July-September 2002.

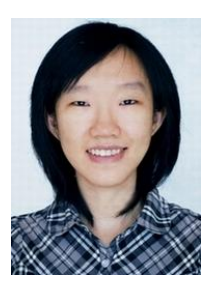

Chao Wang was born in 1987, who pursued her undergraduate years as a major of Applied Mathematics in the Institute of Mathematics Science of Xiamen University and was awarded a B.S. degree from 2005 to 2009.

She is very interested in machine learning technologies, such like support vector machine. During her undergraduate years, she was mainly devoted to the research of the relevant mathematical theories of Clustering Algorithm, and she continued her advanced learning by pursuing a Master's degree in the specialty of computer science.

Currently, she is working and learning in East China Normal University and is mainly engaged in the studies of such relevant fields as computer application technologies and graphic and image processing. In June, 2015, she will be awarded the degree of MS (Master of Science) by East China Normal University. 


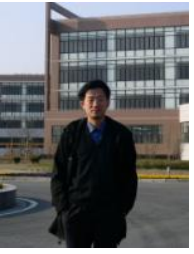

Jun Zheng is a professor of engineer, and the director of Computer Center of East China Normal University, also serving as deputy president of Computation Center (network and modern education) Council at Institute of Shanghai Universities, deputy president at Electrified Education of Shanghai Universities, expert at National Steering Community for Liberal Art Education, and principal of High-performance Computation Center at East China Normal University. His professional emphasis includes research work for WEB application research and high-performance calculations.

He has been occupied in high-performance calculations and R\&D work of computer software for a long time, main partners include Fujitsu Ltd., Yamaha motor group company, NTT DATA and Okasan Securities.

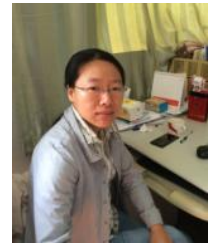

Jianhua Cai was born in Shanghai, China, in 1973. She received the B.S. degree in educational technology in 1996 and the M.S. degree in computer science and technology in 1999 from East China Normal University, Shanghai, China.

She is now a lecturer in the Computer Centre of East China Normal University, who is devoted to teaching and research work of software quality management and software testing. Her professional emphasis includes intelligent optimization Algorithms, image processing and cloud computing. 